\title{
@creative
commons
}

\section{The binary locating-dominating number of some convex polytopes}

\author{
Ana Simić \\ Faculty of Mathematics, University of Belgrade, \\ Studentski trg 16, 11000 Belgrade, Serbia \\ Milena Bogdanović \\ Pedagogical Faculty in Vranje, University of Niš, \\ Partizanska 14, 17500 Vranje, Serbia \\ Jelisavka Milošević \\ FASPER, Visokog Stevana 2, 11000 Belgrade, Serbia
}

Received 11 November 2015, accepted 14 March 2017, published online 23 March 2017

\begin{abstract}
In this paper the binary locating-dominating number of convex polytopes is considered. The exact value is determined and proved for convex polytopes $D_{n}$ and $R_{n}^{\prime \prime}$, while for the convex polytopes $R_{n}, Q_{n}$ and $U_{n}$ a tight upper bound of the locating-dominating number is presented.
\end{abstract}

Keywords: Locating-dominating number, convex polytopes.

Math. Subj. Class.: 05C69, 05C90

\section{Introduction}

Let $G$ be a simple connected undirected graph $G=(V, E)$, where $V$ is a set of vertices, and $E$ is a set of edges. The open neighborhood of a vertex $v \in V$ is $N_{G}(v)=\{u \in V \mid$ $(u, v) \in E\}$ and the closed neighborhood is $N_{G}[v]=\{u \in V \mid(u, v) \in E\} \cup\{v\}$. We write $N(v)$ or $N[v]$ if the graph $G$ is clear from the context [4]. For a graph $G=(V, E)$ a dominating set is a vertex set $D \subseteq V$ such that the union of the closed neighborhoods of the vertices in $D$ is all of $V$; that is, $\bigcup_{v \in D} N[D]=V$. Equivalently, each vertex not in $D$ is adjacent to at least one vertex in $D$, e.g. for every vertex $v \in V \backslash D, N(v) \cap D \neq \emptyset$. The

E-mail addresses: simicana4as@ gmail.com (Ana Simić), mb2001969@beotel.com, milenab@ucfak.ni.ac.rs (Milena Bogdanović), jelisavkam@gmail.com (Jelisavka Milošević) 
domination number of $G$, denoted by $\gamma(G)$, is the minimum cardinality of a dominating set of $G$.

The concept of a dominating set can also be studied through assigning a weight of 1 will be assigned to the vertices in $D$ and a weight of 0 to the vertices of $V \backslash D$. In this case, $D$ is a dominating set of $G$ if for every vertex in $G$ the sum of weights for closed neighborhoods is at least 1, i.e. $|N[v] \cap S| \geq 1$ for each $v \in V$. A dominating set $S \subseteq V$ is a binary locatingdominating set if for every two different vertices $u, v \in V \backslash S$ holds $N(u) \cap S \neq N(v) \cap S$ ([12]). The binary locating-dominating number of $G$, denoted by $\gamma_{l-d}(G)$, is the minimum cardinality of a binary locating-dominating set. In the sequel all terms about the locatingdominating number or set is denoted by binary locating-dominating number or set.

The article [11] studies the smallest cardinalities of locating-dominating codes on chains and cycles and the extreme values of the cardinality of a minimum $r$-identifying or $r$-locating-dominating code in any connected undirected graph $G$ having a given number, $n$, of vertices is studied in [8]. For more information about these issues, see [3, 9, 14, 15, 21]. The authors of the papers [23, 24, 27] study the single-fault-tolerant locating-dominating sets and an open neighborhood locating-dominating sets in trees. More information on locating-dominating sets can be found in [12, 13, 15, 25].

The identifying code problem and binary locating-dominating problem are NP-hard in a general case [6, 7]: I. Charon et al. proved in [7] that, given a graph $G$ and an integer $k$, the decision problem of the existence of an $r$-identifying code, or of an $r$-locating-dominating code, of size at most $k$ in $G$, is NP-complete for any $r$.

The comprehensive list of papers related to identifying code and binary locating-dominating problems were given in [19].

The following theorem gives a tight lower bound of binary locating-dominating number on regular graphs:

Theorem 1.1 (Slater [26]). If $G$ is a regular graph of degree $r$, then

$$
\gamma_{l-d}(G) \geq\left\lceil\frac{2 \cdot|V(G)|}{r+3}\right\rceil .
$$

Graphs of convex polytopes were introduced by Bača [1]. The classes of convex polytopes $Q_{n}$ and $R_{n}$ were introduced in [2]. The metric dimension of convex polytopes $D_{n}$, $Q_{n}$ and $R_{n}$ are equal to 3, as was proved in [16]. In [17] it was proven that metric dimension of convex polytopes $S_{n}, T_{n}$ and $U_{n}$ is also equal to 3 . Minimal doubly resolving sets and the strong metric dimension of convex polytopes $D_{n}$ and $T_{n}$ are studied in [18]. M. Salman et al. [22] were considering three similar optimization problems: the fault-tolerant metric dimension problem, the local metric dimension problem and the strong metric dimension problem of two convex polytopes $S_{n}$ and $U_{n}$.

\section{A modified integer linear programming formulation}

An integer linear programming (ILP) formulation of minimum identifying code problem was given in [5]. If $S$ is an identifying set, then decision variables $x_{i}$ are defined as:

$$
x_{i}= \begin{cases}1, & i \in S \\ 0, & i \notin S\end{cases}
$$

Then, the ILP formulation of minimum identifying code problem from [5] is presented as follows: 


$$
\min \sum_{i \in V} x_{i}
$$

subject to

$$
\begin{array}{cl}
\sum_{j \in N[i]} x_{j} \geq 1, \quad & i \in V \\
\sum_{j \in N[i] \nabla N[k]} x_{j} \geq 1, \quad & i, k \in V, i \neq k \\
x_{i} \in\{0,1\}, \quad & i \in V
\end{array}
$$

The objective function (2.1) ensures that the identifying code set has a minimal cardinality, and constraints (2.2) defines $S$ to be a dominating set. Identifying feature is represented by constraints (2.3) while the binary nature of decision variables $x_{i}$ are given by constraints (2.4).

This formulation can not be directly used for the binary locating-dominating problem. Therefore, it needs to be adapted by changing constraints (2.3) into the constraints (2.5).

$$
x_{i}+x_{k}+\sum_{j \in N(i) \nabla N(k)} x_{j} \geq 1, \quad i, k \in V, i \neq k
$$

Constraints (2.3) and (2.5) are the same when vertices $i$ and $k$ are not neighbors, e.g. $N[i] \nabla N[k]=\{i, j\} \cup(N(i) \nabla N(k))$. The change between (2.3) and (2.5) is reflected only when vertices $i$ and $k$ are neighbors, i.e. $i \in N(k)$. Then, by constraints (2.5), at least one of vertices $i, k$ or some $j \in N(i) \nabla N(k)$ must be in $S$. When $i$ and $k$ are not neighbors, then $N[i] \nabla N[k]=\{i, j\} \cup(N(i) \nabla N(k))$, so constraints (2.3) and (2.5) are equal.

In [28] it was noted that if $d(u, v) \geq 3$ then $u, v$ has no neighbors in common, therefore, $N(u) \cap S \neq N(v) \cap S$ need not be checked for equivalence. This becomes computationally important for large graphs as it allows us to minimize the number of constraints generated by the locating requirement. Using this idea, constraints (2.5) would be further improved:

$$
x_{i}+x_{k}+\sum_{j \in N(i) \nabla N(k)} x_{j} \geq 1, \quad i, k \in V, i \neq k, d(i, k) \leq 2
$$

The proposed formulation with a reduced number of constraints can be used to find the exact optimal values for problems of small dimensions. Moreover, as it can be seen from [10], ILP formulation can be tackled by efficient metaheuristic approaches for obtaining suboptimal solutions for large dimensions.

\section{The exact values}

\subsection{Convex polytope $D_{n}$}

The graph of convex polytope $D_{n}$, on Figure 1, was introduced in [16]. It consists of $2 n$ 5-sided faces and a pair of $n$-sided faces. Mathematically, it has vertex set $V\left(D_{n}\right)=$ $\left\{a_{i}, b_{i}, c_{i}, d_{i} \mid i=0,1, \ldots, n-1\right\}$ and edge set $E\left(D_{n}\right)=\left\{\left(a_{i}, a_{i+1}\right),\left(d_{i}, d_{i+1}\right),\left(a_{i}, b_{i}\right)\right.$, $\left.\left(b_{i}, c_{i}\right),\left(c_{i}, d_{i}\right),\left(b_{i+1}, c_{i}\right) \mid i=0,1, \ldots, n-1\right\}$. Note that arithmetic in the subscripts is performed modulo $n$. 


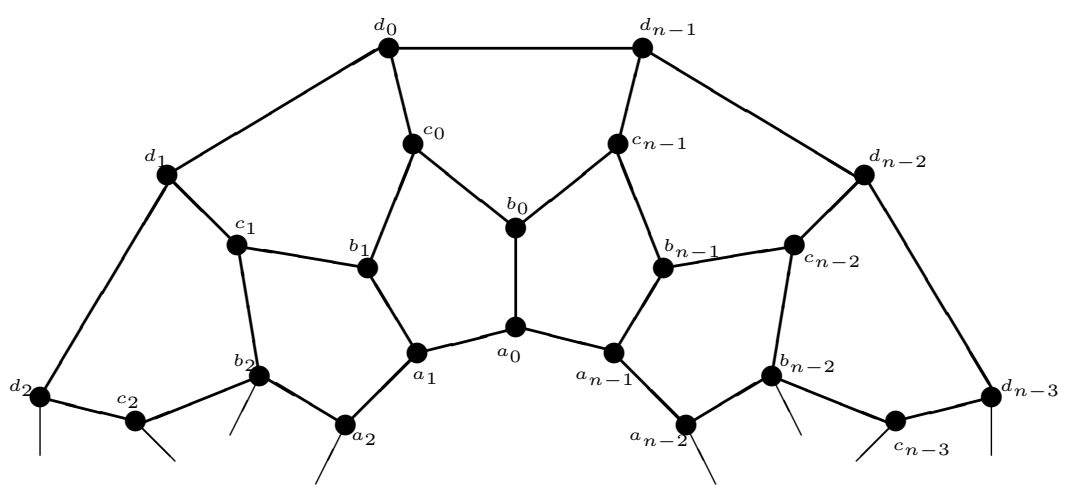

Figure 1: The graph of convex polytope $D_{n}$.

Table 1: Locating-dominating vertices in $D_{n}$.

\begin{tabular}{|l|c|c|c|c|}
\hline$n$ & $v \in V \backslash S$ & $S \bigcap N[v]$ & $v \in V \backslash S$ & $S \bigcap N[v]$ \\
\hline $3 k$ & $a_{3 i}$ & $\left\{a_{3 i+1}, b_{3 i}\right\}$ & $a_{3 i+2}$ & $\left\{a_{3 i+1}\right\}$ \\
& $b_{3 i+1}$ & $\left\{a_{3 i+1}, c_{3 i+1}\right\}$ & $b_{3 i+2}$ & $\left\{c_{3 i+1}\right\}$ \\
& $c_{3 i}$ & $\left\{b_{3 i}\right\}$ & $c_{3 i+2}$ & $\left\{b_{3(i+1)}, d_{3 i+2}\right\}$ \\
& $d_{3 i}$ & $\left\{d_{3(i-1)+2}\right\}$ & $d_{3 i+1}$ & $\left\{c_{3 i+1}, d_{3 i+2}\right\}$ \\
\hline $3 k+1$ & $a_{3 i}$ & $\left\{a_{3(i-1)+2}, b_{3 i}\right\}$ & $a_{3 i+1}$ & $\left\{a_{3 i+2}\right\}$ \\
& $b_{3 i+1}$ & $\left\{c_{3 i+1}\right\}$ & $b_{3 i+2}$ & $\left\{a_{3 i+2}, c_{3 i+1}\right\}$ \\
& $c_{3 i}$ & $\left\{b_{3 i}, d_{3 i}\right\}$ & $c_{3 i+2}$ & $\left\{b_{3(i+1)}\right\}$ \\
& $d_{3 i+1}$ & $\left\{c_{3 i+1}, d_{3 i}\right\}$ & $d_{3 i+2}$ & $\left\{d_{3(i+1)}\right\}$ \\
& $a_{3 k}$ & $\left\{b_{3 k}, a_{3(k-1)+2}\right\}$ & $c_{3 k}$ & $\left\{b_{3 k}, d_{3 k}\right\}$ \\
& $a_{0}$ & $\left\{b_{0}\right\}$ & & \\
\hline $3 k+2$ & $a_{3 i+1}$ & $\left\{a_{3 i}, b_{3 i+1}\right\}$ & $a_{3 i+2}$ & $\left\{a_{3(i+1)}\right\}$ \\
& $b_{3 i}$ & $\left\{a_{3 i}, c_{3(i-1)+2}\right\}$ & $b_{3 i+2}$ & $\left\{c_{3 i+2}\right\}$ \\
& $c_{3 i}$ & $\left\{b_{3 i+1}, d_{3 i}\right\}$ & $c_{3 i+1}$ & $\left\{b_{3 i+1}\right\}$ \\
& $d_{3 i+1}$ & $\left\{d_{3 i}\right\}$ & $d_{3 i+2}$ & $\left\{c_{3 i+2}, d_{3(i+1)}\right\}$ \\
& $b_{3 k}$ & $\left\{a_{3 k}, c_{3(k-1)+2}\right\}$ & $c_{3 k}$ & $\left\{b_{3 k+1}, d_{3 k}\right\}$ \\
& $a_{3 k+1}$ & $\left\{a_{3 k}, b_{3 k+1}\right\}$ & $c_{3 k+1}$ & $\left\{b_{3 k+1}\right\}$ \\
& $d_{3 k+1}$ & $\left\{d_{3 k}\right\}$ & $b_{0}$ & $\left\{a_{0}\right\}$ \\
\hline
\end{tabular}




\section{Theorem 3.1.}

$$
\gamma_{l-d}\left(D_{n}\right)=\left\lceil\frac{4 \cdot n}{3}\right\rceil .
$$

Proof. Firstly, notice that $D_{n}$ is a regular graph of degree 3, with $4 n$ vertices. Then, by Theorem 1.1 it holds $\gamma_{l-d}\left(D_{n}\right) \geq\left\lceil\frac{2 \cdot 4 \cdot n}{3+3}\right\rceil=\left\lceil\frac{4 \cdot n}{3}\right\rceil$.

Let

$$
S= \begin{cases}\left\{a_{3 i+1}, b_{3 i}, c_{3 i+1}, d_{3 i+2} \mid i=0, \ldots, k-1\right\}, & n=3 k \\ \left\{b_{3 k}, d_{3 k}\right\} \bigcup\left\{a_{3 i+2}, b_{3 i}, c_{3 i+1}, d_{3 i} \mid i=0, \ldots, k-1\right\}, & n=3 k+1 \\ \left\{a_{3 k}, b_{3 k+1}, d_{3 k}\right\} \bigcup\left\{a_{3 i}, b_{3 i+1}, c_{3 i+2}, d_{3 i} \mid i=0, \ldots, k-1\right\}, & n=3 k+2\end{cases}
$$

Now, let us prove that $S$ is a locating-dominating set of $D_{n}$. In order to do that, we need to consider three possible cases:

Case 1: $n=3 k$. As can be seen from Table 1, neighborhoods of all vertices in $V \backslash S$ and their intersections with set $S$ are non-empty and distinct. Although some formulas for some intersections can be somewhat similar, they are distinct. For example, $S \bigcap N\left[a_{3 i+2}\right]=\left\{a_{3(i+1)}\right\} \neq\left\{a_{3 i+1}\right\}=S \bigcap N\left[b_{3 i+1}\right]$, since indices $3(i+1)=$ $3 i+3 \neq 3 i+1$. Similarly, $S \bigcap N\left[d_{3 i+1}\right]=\left\{c_{3 i+2}, d_{3 i}\right\} \neq\left\{c_{3 i+2}, d_{3(i+1)}\right\}=$ $S \cap N\left[d_{3 i+2}\right]$;

Case 2: $n=3 k+1$. As in the previous case, once again, all intersections of neighborhoods $N[v]$ with set $S$, i.e. $S \bigcap N[v]$, are non-empty and distinct. This also can be seen from Table 1;

Case 3: $n=3 k+2$. As in both previous cases, once again, all intersections of neighborhoods $N[v]$ with set $S$, i.e. $S \bigcap N[v]$, are non-empty and distinct, which also can be seen from Table 1.

\subsection{Convex polytope $R_{n}^{\prime \prime}$}

The graph of convex polytope $R_{n}^{\prime \prime}$ on Figure 2 is introduced in [20]. It has vertex set $V=\left\{a_{i}, b_{i}, c_{i}, d_{i}, e_{i}, f_{i} \mid i=0, \ldots, n-1\right\}$ and edge set $E=\left\{\left(a_{i}, a_{i+1}\right),\left(a_{i}, b_{i}\right),\left(b_{i}, c_{i}\right)\right.$, $\left.\left(b_{i+1}, c_{i}\right),\left(c_{i}, d_{i}\right),\left(d_{i}, e_{i}\right),\left(d_{i+1}, e_{i}\right),\left(e_{i}, f_{i}\right),\left(f_{i}, f_{i+1}\right) \mid i=0, \ldots, n-1\right\}$.

\section{Theorem 3.2.}

$$
\gamma_{l-d}\left(R_{n}^{\prime \prime}\right)=2 \cdot n
$$

Proof. It can be seen that $R_{n}^{\prime \prime}$ is a regular graph of degree 3, with $6 n$ vertices. Then, by Theorem 1.1 it holds $\gamma_{l-d}\left(R_{n}^{\prime \prime}\right) \geq\left[\frac{2 \cdot 6 \cdot n}{3+3}\right]=2 \cdot n$. Now, let us prove that a set $S=\left\{b_{i}, e_{i} \mid\right.$ $i=0, \ldots, n-1\}$ is a binary locating-dominating set of $R_{n}^{\prime \prime}$. Indeed, it is easy to see that all intersections $S \bigcap N\left[a_{i}\right]=\left\{b_{i}\right\} ; S \bigcap N\left[c_{i}\right]=\left\{b_{i}, b_{i+1}\right\} ; S \bigcap N\left[d_{i}\right]=\left\{e_{i-1}, e_{i}\right\}$ and $S \bigcap N\left[f_{i}\right]=\left\{e_{i}\right\}$ are non-empty and distinct. Since $S$ is a binary locating-dominating set of $R_{n}^{\prime \prime}$ and $|S|=2 \cdot n$ therefore, $\gamma_{l-d}\left(R_{n}^{\prime \prime}\right) \leq 2 \cdot n$. Due to the previously proved fact that $\gamma_{l-d}\left(R_{n}^{\prime \prime}\right) \geq 2 \cdot n$, it is proven that $\gamma_{l-d}\left(R_{n}^{\prime \prime}\right)$ is equal to $2 \cdot n$.

\section{The upper bounds}

\subsection{Convex polytope $Q_{n}$}

The graph of convex polytope $Q_{n}$ in Figure 3, is introduced in [2]. It has vertex set $V\left(Q_{n}\right)=\left\{a_{i}, b_{i}, c_{i}, d_{i} \mid i=0,1, \ldots, n-1\right\}$ and edge set $E\left(Q_{n}\right)=\left\{\left(a_{i}, a_{i+1}\right),\left(b_{i}, b_{i+1}\right)\right.$, 


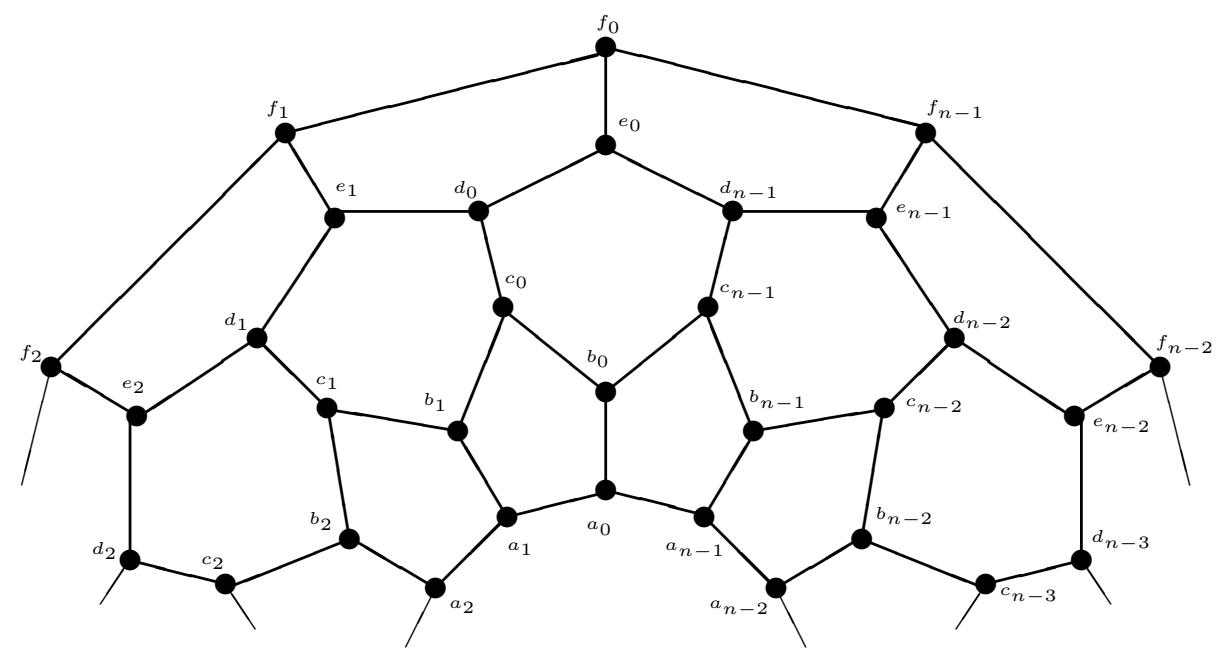

Figure 2: The graph of convex polytope $R_{n}^{\prime \prime}$.

$\left.\left(d_{i}, d_{i+1}\right),\left(a_{i}, b_{i}\right),\left(b_{i}, c_{i}\right),\left(c_{i}, d_{i}\right),\left(b_{i+1}, c_{i}\right) \mid i=0,1, \ldots, n-1\right\}$. We call the cycle induced by set of vertices $\left\{a_{0}, a_{1}, \ldots, a_{n-1}\right\}$ the inner cycle, the cycle induced by $\left\{d_{0}, d_{1}, \ldots, d_{n-1}\right\}$ the outer cycle, and the middle cycle are induced by set of vertices $\left\{b_{0}, b_{1}, \ldots, b_{n-1}\right\}$. This polytope consists of $n$-sided faces, $n 4$-sided faces and $n$ triangles.

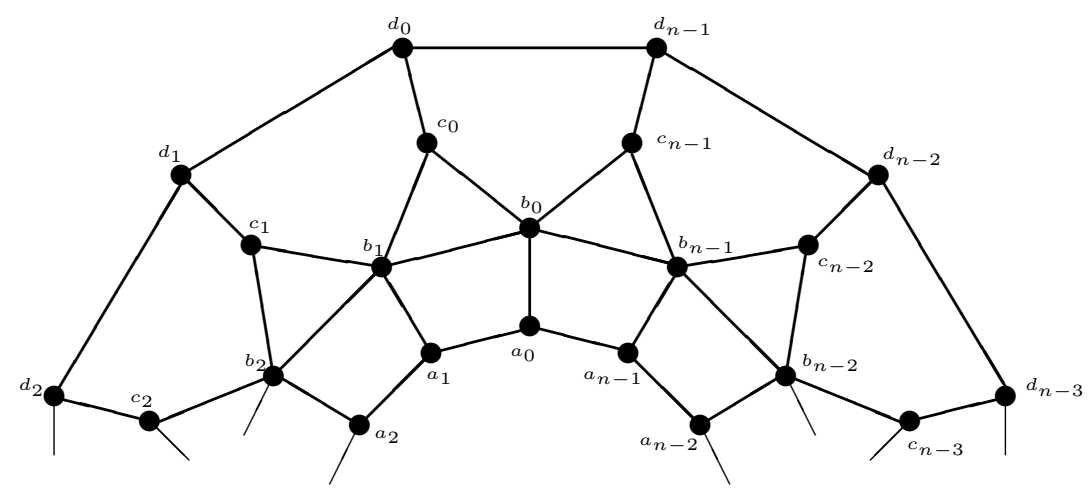

Figure 3: The graph of convex polytope $Q_{n}$.

\section{Theorem 4.1.}

$$
\gamma_{l-d}\left(Q_{n}\right) \leq\left\lceil\frac{4 \cdot n}{3}\right\rceil
$$

and this bound is tight. 
Table 2: Additional data for $Q_{n}$ compared to $D_{n}$.

\begin{tabular}{|l|c|c|c|c|}
\hline$n$ & $v \in V \backslash S$ & $S \bigcap N[v]$ & $v \in V \backslash S$ & $S \bigcap N[v]$ \\
\hline $3 k$ & $b_{3 i+1}$ & $\left\{a_{3 i+1}, b_{3 i}, c_{3 i+1}\right\}$ & $b_{3 i+2}$ & $\left\{b_{3(i+1)}, c_{3 i+1}\right\}$ \\
\hline $3 k+1$ & $b_{3 i+1}$ & $\left\{b_{3 i}, c_{3 i+1}\right\}$ & $b_{3 i+2}$ & $\left\{a_{3 i+2}, b_{3(i+1)}, c_{3 i+1}\right\}$ \\
\hline $3 k+2$ & $b_{3 i}$ & $\left\{a_{3 i}, b_{3 i+1}, c_{3(i-1)+2}\right\}$ & $b_{3 i+2}$ & $\left\{b_{3 i+1}, c_{3 i+2}\right\}$ \\
& $b_{3 k}$ & $\left\{a_{3 k}, b_{3 k+1}, c_{3(k-1)+2}\right\}$ & $b_{0}$ & $\left\{a_{0}, b_{1}, b_{3 k+1}\right\}$ \\
\hline
\end{tabular}

Proof. Let

$$
S= \begin{cases}\left\{a_{3 i+1}, b_{3 i}, c_{3 i+1}, d_{3 i+2} \mid i=0, \ldots, k-1\right\}, & n=3 k \\ \left\{b_{3 k}, d_{3 k}\right\} \bigcup\left\{a_{3 i+2}, b_{3 i}, c_{3 i+1}, d_{3 i} \mid i=0, \ldots, k-1\right\}, & n=3 k+1 \\ \left\{a_{3 k}, b_{3 k+1}, d_{3 k}\right\} \bigcup\left\{a_{3 i}, b_{3 i+1}, c_{3 i+2}, d_{3 i} \mid i=0, \ldots, k-1\right\}, & n=3 k+2\end{cases}
$$

Note that this set is the same as for convex polytopes $D_{n}$. This is not a surprise, since convex polytopes $Q_{n}$ have only $n$ additional edges $\left(b_{i}, b_{i+1}\right), i=0, \ldots, n-1$ compared to $D_{n}$. Therefore, except vertices $b_{i}, i=0, \ldots, n-1$, all neighborhoods of vertices in $V \backslash S$ and their intersection with set $S$ are the same as in Table 1. Additional data is presented in Table 2.

As can be seen from Table 3 and additional data from Table 2, in all three cases, neighborhoods of all vertices in $V \backslash S$ and their intersection with set $S$ are non-empty and distinct. Therefore set $S$ is a locating-dominating set for $Q_{n}$. Since $|S|=\left\lceil\frac{4 \cdot n}{3}\right\rceil$ therefore, $\gamma_{l-d}\left(Q_{n}\right) \leq\left\lceil\frac{4 \cdot n}{3}\right\rceil$.

Using the CPLEX solver on the integer linear programming formulation (2.1), (2.2), (2.4), and (2.6) we have obtained optimal solutions: $\gamma_{l-d}\left(Q_{5}\right)=7, \gamma_{l-d}\left(Q_{6}\right)=8$, $\gamma_{l-d}\left(Q_{7}\right)=10, \ldots, \gamma_{l-d}\left(Q_{28}\right)=38, \gamma_{l-d}\left(Q_{29}\right)=39$ and $\gamma_{l-d}\left(Q_{30}\right)=40$ which all match the proposed upper bound in this theorem. Therefore, the proposed upper bound is tight.

\subsection{Convex polytope $R_{n}$}

The graph of convex polytope $R_{n}$, on Figure 4, has been introduced in [2]. It has vertex set $V=\left\{a_{i}, b_{i}, c_{i} \mid i=0, \ldots, n-1\right\}$ and edge set $E=\left\{\left(a_{i}, a_{i+1}\right),\left(a_{i}, b_{i}\right),\left(a_{i+1}, b_{i}\right)\right.$, $\left.\left(b_{i}, b_{i+1}\right),\left(b_{i}, c_{i}\right),\left(c_{i}, c_{i+1}\right) \mid i=0, \ldots, n-1\right\}$. This graph consists of $n$ 4-sided faces and $2 n$ triangles.

\section{Theorem 4.2.}

$$
\gamma_{l-d}\left(R_{n}\right) \leq n
$$

and this bound is tight.

Proof. Let $S=\left\{b_{i} \mid i=0, \ldots, n-1\right\}$. It is easy to see that all intersections $S \cap N\left[a_{i}\right]=$ $\left\{b_{i-1}, b_{i}\right\}$ and $S \bigcap N\left[c_{i}\right]=\left\{b_{i}\right\}$ are non-empty and distinct. Since $S$ is a binary locatingdominating set of $R_{n}$ and $|S|=n$ therefore, $\gamma_{l-d}\left(R_{n}\right) \leq n$.

Using the CPLEX solver on integer linear programming formulation (2.1), (2.2), (2.4), and (2.6), we have obtained optimal solutions. For $5 \leq n \leq 31, \gamma_{l-d}\left(R_{n}\right)=n$, which match the proposed upper bound in this theorem. Therefore, the proposed upper bound is tight. 


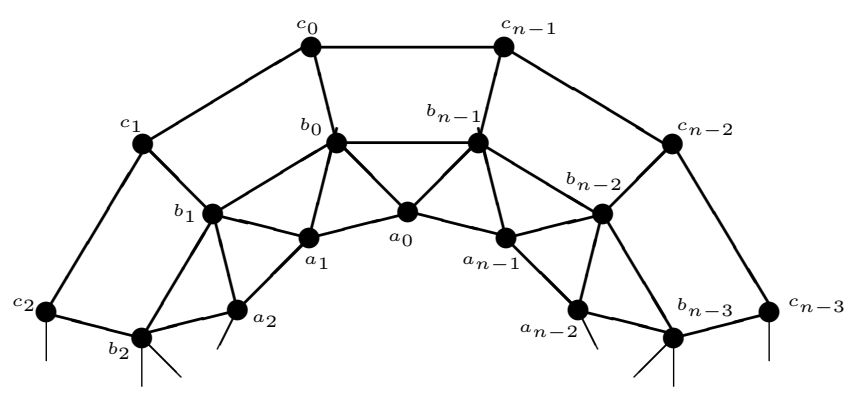

Figure 4: The graph of convex polytope $R_{n}$.

\subsection{Convex polytope $U_{n}$}

Mathematically, the graph of convex polytope $U_{n}$, on Figure 5, introduced in [17], has vertex set $V=\left\{a_{i}, b_{i}, c_{i}, d_{i}, e_{i} \mid i=0, \ldots, n-1\right\}$ and edge set $E=\left\{\left(a_{i}, a_{i+1}\right),\left(a_{i}, b_{i}\right)\right.$, $\left.\left(b_{i}, b_{i+1}\right),\left(b_{i}, c_{i}\right),\left(c_{i}, d_{i}\right),\left(c_{i+1}, d_{i}\right),\left(d_{i}, e_{i}\right),\left(e_{i}, e_{i+1}\right) \mid i=0, \ldots, n-1\right\}$. This graph in Figure 5 has $2 n$-sided faces and $n$ 4-sided faces.

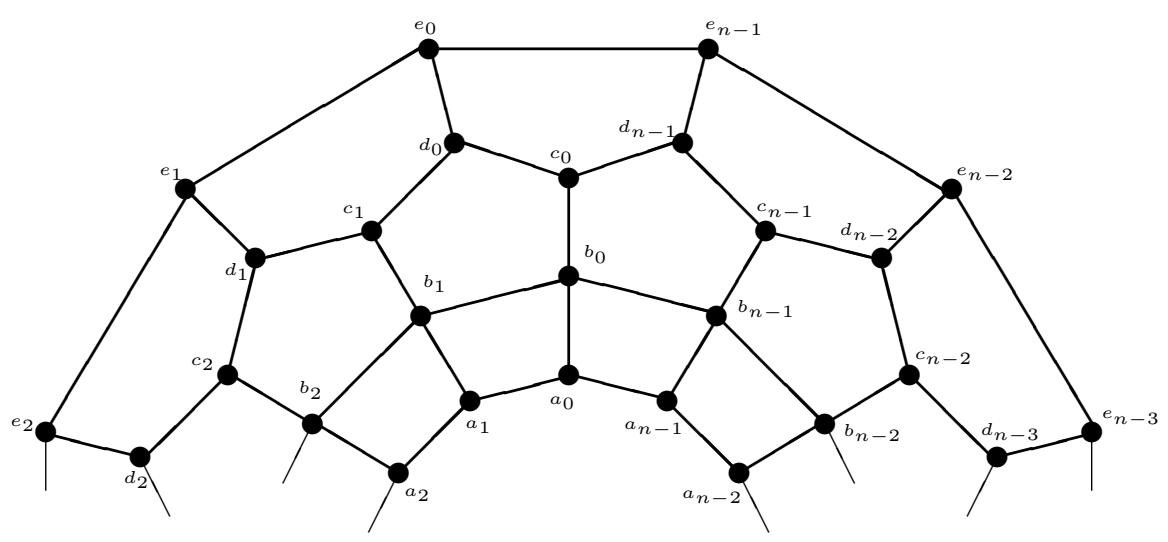

Figure 5: The graph of convex polytope $U_{n}$.

\section{Theorem 4.3.}

$$
\gamma_{l-d}\left(U_{n}\right) \leq\left\lceil\frac{5 \cdot n}{3}\right\rceil
$$

and this bound is tight.

Proof. If $n=3 k$, let $S=\left\{a_{3 i+1}, b_{3 i}, c_{3 i+1}, d_{3 i+2}, e_{3 i} \mid i=0, \ldots, k-1\right\}$, if $n=3 k+1$ let $S=\left\{a_{3 k}, c_{3 k}, e_{3 k}\right\} \bigcup\left\{a_{3 i}, b_{3 i+2}, c_{3 i}, d_{3 i+1}, e_{3 i} \mid i=0, \ldots, k-1\right\}$ and if $n=3 k+2$ let $S=\left\{a_{3 k+1}, b_{3 k}, c_{3 k+1}, e_{3 k+1}\right\} \bigcup\left\{a_{3 i+1}, b_{3 i}, c_{3 i+1}, d_{3 i+2}, e_{3 i+1} \mid i=0, \ldots, k-1\right\}$.

Now, let us prove that $S$ is a locating-dominating set of $U_{n}$. In order to do that, as we did in proofs of previous Theorems, we need to consider three possible cases. As it can be seen from Table 3, in all three cases, neighborhoods of all vertices in $V \backslash S$ and their intersection with set $S$ are non-empty and distinct. 
Using the CPLEX solver on the integer linear programming formulation (2.1), (2.2), (2.4), and (2.6) we have obtained optimal solutions: $\gamma_{l-d}\left(U_{5}\right)=9, \gamma_{l-d}\left(U_{6}\right)=10$, $\gamma_{l-d}\left(U_{7}\right)=12, \ldots, \gamma_{l-d}\left(U_{22}\right)=38, \gamma_{l-d}\left(U_{23}\right)=39$ and $\gamma_{l-d}\left(U_{24}\right)=40$ which all match the proposed upper bound in this theorem. Therefore, the proposed upper bound is tight.

Table 3: Locating-dominating vertices in $U_{n}$.

\begin{tabular}{|l|c|c|c|c|}
\hline$n$ & $v \in V \backslash S$ & $S \cap N[v]$ & $v \in V \backslash S$ & $S \cap N[v]$ \\
\hline $3 k$ & $a_{3 i}$ & $\left\{a_{3 i+1}, b_{3 i}\right\}$ & $a_{3 i+2}$ & $\left\{a_{3 i+1)}\right\}$ \\
& $b_{3 i+1}$ & $\left\{a_{3 i+1}, b_{3 i}, c_{3 i+1}\right\}$ & $b_{3 i+2}$ & $\left\{b_{3(i+1)}\right\}$ \\
& $c_{3 i}$ & $\left\{b_{3 i}, d_{3(i-1)+2}\right\}$ & $c_{3 i+2}$ & $\left\{d_{3 i+2}\right\}$ \\
& $d_{3 i}$ & $\left\{c_{3 i+1}, e_{3 i}\right\}$ & $d_{3 i+1}$ & $\left\{c_{3 i+1}\right\}$ \\
& $e_{3 i+1}$ & $\left\{e_{3 i}\right\}$ & $e_{3 i+2}$ & $\left\{d_{3 i+2}, e_{3(i+1)}\right\}$ \\
\hline $3 k+1$ & $a_{3 i+1}$ & $\left\{a_{3 i}\right\}$ & $a_{3 i+2}$ & $\left\{a_{3(i+1)}, b_{3 i+2}\right\}$ \\
& $b_{3 i}$ & $\left\{a_{3 i}, b_{3(i-1)+2}, c_{3 i}\right\}$ & $b_{3 i+1}$ & $\left\{b_{3 i+2}\right\}$ \\
& $c_{3 i+1}$ & $\left\{d_{3 i+1}\right\}$ & $c_{3 i+2}$ & $\left\{b_{3 i+2}, d_{3 i+1}\right\}$ \\
& $d_{3 i}$ & $\left\{c_{3 i}, e_{3 i}\right\}$ & $d_{3 i+2}$ & $\left\{c_{3(i+1)}\right\}$ \\
& $e_{3 i+1}$ & $\left\{d_{3 i+1}, e_{3 i}\right\}$ & $e_{3 i+2}$ & $\left\{e_{3(i+1)}\right\}$ \\
& $b_{3 k}$ & $\left\{a_{3 k}, b_{3(k-1)+2}, c_{3 k}\right\}$ & $d_{3 k}$ & $\left\{c_{3 k}, e_{3 k}\right\}$ \\
& $b_{0}$ & $\left\{a_{0}, c_{0}\right\}$ & & \\
\hline $3 k+2$ & $a_{3 i}$ & $\left\{a_{3 i+1}, b_{3 i}\right\}$ & $a_{3 i+2}$ & $\left\{a_{3 i+1}\right\}$ \\
& $b_{3 i+1}$ & $\left\{a_{3 i+1}, b_{3 i}, c_{3 i+1}\right\}$ & $b_{3 i+2}$ & $\left\{b_{3 i+1}\right\}$ \\
& $c_{3 i}$ & $\left\{b_{3 i}, d_{3(i-1)+2}\right\}$ & $c_{3 i+2}$ & $\left\{d_{3 i+2}\right\}$ \\
& $d_{3 i}$ & $\left\{c_{3 i+1}\right\}$ & $d_{3 i+1}$ & $\left\{c_{3 i+1}, e_{3 i+1}\right\}$ \\
& $e_{3 i}$ & $\left\{e_{3 i+1}\right\}$ & $e_{3 i+2}$ & $\left\{d_{3 i+2}, e_{3 i+1}\right\}$ \\
& $a_{3 k}$ & $\left\{a_{3 k+1}, b_{3 k}\right\}$ & $c_{3 k}$ & $\left\{b_{3 k}, d_{3(k-1)+2}\right\}$ \\
& $d_{3 k}$ & $\left\{c_{3 k+1}\right\}$ & $e_{3 k}$ & $\left\{e_{3 k+1}\right\}$ \\
& $b_{3 k+1}$ & $\left\{a_{3 k+1}, b_{0}, b_{3 k}, c_{3 k+1}\right\}$ & $d_{3 k+1}$ & $\left\{c_{3 k+1}, e_{3 k+1}\right\}$ \\
& $a_{0}$ & $\left\{a_{1}, a_{3 k+1}, b_{0}\right\}$ & $c_{0}$ & $\left\{b_{0}\right\}$ \\
& $e_{0}$ & $\left\{e_{1}, e_{3 k+1}\right\}$ & & \\
\hline
\end{tabular}

\section{Conclusions}

In this paper, we are studying the locating-dominating sets and the binary locating-dominating number of some convex polytopes. We are dealing with some classes of convex polytopes by considering classes: $D_{n}, R_{n}^{\prime \prime}, R_{n}, Q_{n}$ and $U_{n}$. For $D_{n}$ and $R_{n}^{\prime \prime}$ exact values are obtained and proved, while for $R_{n}, Q_{n}$ and $U_{n}$ tight upper bounds are given.

Future work can be directed towards determining a binary locating-dominating set of some other challenging classes of graphs. The other promising direction for future work is solving of some other similar graph problem on convex polytopes.

\section{References}

[1] M. Bača, Labelings of two classes of convex polytopes, Utilitas Math. 34 (1988), 24-31.

[2] M. Bača, On magic labellings of convex polytopes, Ann. Discrete Math. 51 (1992), 13-16, doi:10.1016/s0167-5060(08)70599-5. 
[3] C. Balbuena, F. Foucaud and A. Hansberg, Locating-dominating sets and identifying codes in graphs of girth at least 5, Electron. J. Combin. 22 (2015), \#P2.15, http://www. combinatorics.org/ojs/index.php/eljc/article/view/v22i2p15.

[4] D. W. Bange, A. E. Barkauskas, L. H. Host and P. J. Slater, Generalized domination and efficient domination in graphs, Discrete Math 159 (1996), 1-11, doi:10.1016/0012-365x(95) 00094-d.

[5] N. Bousquet, A. Lagoutte, Z. Li, A. Parreau and S. Thomassé, Identifying codes in hereditary classes of graphs and VC-dimension, SIAM J. Discrete Math. 29 (2015), 2047-2064, doi:10. 1137/14097879x.

[6] I. Charon, O. Hudry and A. Lobstein, Identifying and locating-dominating codes: NPcompleteness results for directed graphs, IEEE Trans. Inform. Theory 48 (2002), 2192-2200, doi:10.1109/tit.2002.800490.

[7] I. Charon, O. Hudry and A. Lobstein, Minimizing the size of an identifying or locatingdominating code in a graph is NP-hard, Theor. Comput. Sci. 290 (2003), 2109-2120, doi: 10.1016/s0304-3975(02)00536-4.

[8] I. Charon, O. Hudry and A. Lobstein, Extremal cardinalities for identifying and locatingdominating codes in graphs, Discrete Math. 307 (2007), 356-366, doi:10.1016/j.disc.2005. 09.027 .

[9] C. Chen, C. Lu and Z. Miao, Identifying codes and locating-dominating sets on paths and cycles, Discrete Appl. Math. 159 (2011), 1540-1547, doi:10.1016/j.dam.2011.06.008.

[10] S. Hanafi, J. Lazić, N. Mladenović and I. Wilbaut, C.and Crévits, New variable neighbourhood search based 0-1 mip heuristics, Yugosl. J. Oper. Res. 25 (2015), 343-360, doi: 10.2298/yjor140219014h.

[11] T. W. Haynes, M. A. Henning and J. Howard, Locating and total dominating sets in trees, Discrete Appl. Math. 154 (2006), 1293-1300, doi:10.1016/j.dam.2006.01.002.

[12] C. Hernando, M. Mora and I. M. Pelayo, LD-graphs and global location-domination in bipartite graphs, Electron. Notes Discrete Math. 46 (2014), 225-232, doi:10.1016/j.endm.2014.08.030.

[13] I. Honkala, An optimal locating-dominating set in the infinite triangular grid, Discrete Math. 306 (2006), 2670-2681, doi:10.1016/j.disc.2006.04.028.

[14] I. Honkala, O. Hudry and A. Lobstein, On the ensemble of optimal dominating and locatingdominating codes in a graph, Inform. Process. Lett. 115 (2015), 699-702, doi:10.1016/j.ipl. 2015.04.005.

[15] I. Honkala and T. Laihonen, On locating-dominating sets in infinite grids, European J. Combin. 27 (2006), 218-227, doi:10.1016/j.ejc.2004.09.002.

[16] M. Imran, A. Q. Baig and A. Ahmad, Families of plane graphs with constant metric dimension, Utilitas Math. 88 (2012), 43-57.

[17] M. Imran, S. A. U. H. Bokhary and A. Q. Baig, On families of convex polytopes with constant metric dimension, Comput. Math. Appl. 60 (2010), 2629-2638, doi:10.1016/j.camwa.2010.08. 090 .

[18] J. Kratica, V. Kovačević-Vujčić, M. Čangalović and M. Stojanović, Minimal doubly resolving sets and the strong metric dimension of some convex polytopes, Appl. Math. Comput. 218 (2012), 9790-9801, doi:10.1016/j.amc.2012.03.047.

[19] A. Lobstein, Watching systems, identifying, locating-dominating and discriminating codes in graphs, http://perso.telecom-paristech.fr/ lobstein/ debutBIBidetlocdom.pdf, a bibliography. 
[20] M. Miller, M. Bača and J. A. MacDougall, Vertex-magic total labeling of generalized Petersen graphs and convex polytopes, J. Combin. Math. Combin. Comput. 59 (2006), 89-99, http: //www. combinatorialmath.ca/ jcmec/ jcmec59.html.

[21] T. Müller and J.-S. Sereni, Identifying and locating-dominating codes in (random) geometric networks, Combin. Probab. Comput. 18 (2009), 925-952, doi:10.1017/s0963548309990344.

[22] M. Salman, I. Javaid and M. A. Chaudhry, Minimum fault-tolerant, local and strong metric dimension of graphs, 2014, arXiv:1409.2695 [math.CO].

[23] S. J. Seo and P. J. Slater, Open neighborhood locating-dominating sets, Australas. J. Combin. 46 (2010), 109-119, https: / / a jc.maths.uq.edu.au/pdf/46/ajc_v46_p109. pdf.

[24] S. J. Seo and P. J. Slater, Open neighborhood locating-dominating in trees, Discrete Appl. Math. 159 (2011), 484-489, doi:10.1016/j.dam.2010.12.010.

[25] P. J. Slater, Domination and location in acyclic graphs, Networks 17 (1987), 55-64, doi:10. 1002/net.3230170105.

[26] P. J. Slater, Locating dominating sets and locating-dominating sets, in: Y. Alavi and A. Schwenk (eds.), Graph Theory, Combinatorics, and Algorithms, John Wiley \& Sons, New York, volume 2, pp. 1073-1079, 1995, proceedings of the Seventh Quadrennial International Conference on the Theory and Applications of Graphs, Western Michigan University.

[27] P. J. Slater, Fault-tolerant locating-dominating sets, Discrete Math. 249 (2002), 179-189, doi: 10.1016/s0012-365x(01)00244-8.

[28] D. B. Sweigart, J. Presnell and R. Kincaid, An integer program for open locating dominating sets and its results on the hexagon-triangle infinite grid and other graphs, in: 2014 Systems and Information Engineering Design Symposium (SIEDS), Charlottesville, Virginia, 2014 pp. 29-32, doi:10.1109/sieds.2014.6829887. 